\title{
A comprehensive study on the stability analysis of vehicle dynamics with pure/combined slip tire models
}

\author{
Ehsan Hashemi, Mohammad Pirani, Amir Khajepour, and Alireza Kasaiezadeh
}

\begin{abstract}
In this paper, a vehicle's lateral dynamic model is developed based on the pure and the combined-slip LuGre tire models. Conventional vehicle's lateral dynamic methods derive handling models utilizing linear tires and pure-slip assumptions. The current article proposes a general lateral dynamic model, which takes the linear and nonlinear behaviors of the tire into account using the pure and combined-slip assumptions separately. The developed methodology also incorporates various normal loads at each corner and provides a proper tire-vehicle platform for control and estimation applications. Steady-state and transient LuGre models are also used in the model development and their responses are compared in different driving scenarios. Considering the fact that the vehicle dynamics is time-varying, the stability of the suggested time-varying model is investigated using an affine quadratic stability approach, and a novel approach to define the critical longitudinal speed is suggested and compared with that of conventional lateral stability methods. Simulations have been conducted and the results are used to validate the proposed method.
\end{abstract}

\section{INTRODUCTION}

Tire models and tire-road friction forces have played a vital role in recent developments in the field of vehicle state estimation and control. They are incorporated into the lateral dynamics to estimate vehicle states and to analyze stability. The most widely used static tire model, known as the Magic Formula, was proposed by Pacejka et al. [1], [2], and Uil [3], and provides a semi-experimental approach for tire force calculation. Canudas-de-Wit et al. proposed a dynamic tire-road friction model, known as the LuGre, in [4]-[8], and introduced tire deflection as a state in system dynamics. Pre-sliding and hysteresis loops as well as combined friction characteristics are considered in their model [9]. Other dynamic models, examined in [10] and [11], consider transient phases.

In the vehicle stability analysis, the most widely practiced approach uses a 2-degree-of-freedom (2DOF) planar vehicle model and a linear tire model [12], [13]. These studies fail to predict the critical velocity and under/neutral/over steering behavior of the actual vehicle in nonlinear operating regions.

Vincent Nguyen [14] has studied the stability of vehicle lateral dynamics with bifurcation of the equilibrium conditions, and also explored some aspects of the transient response of vehicles. The study used a planar model with roll dynamics, lateral load transfer, and tire dynamics for overall stability assessment outside of normal operating ranges. Different tire models and their associated friction situations in antilock brake systems (ABSs) and longitudinal dynamics are discussed in [15]. Tire forces were used as a cascaded scheme for longitudinal vehicle dynamics, but a closed formulation of the integrated tire-vehicle with a dynamic tire model, such as LuGre [6], is not provided.

A theoretical analysis followed by a numerical study was performed in [16] and [17] to develop a new methodology for the local stability assessment of vehicle dynamics. The effects of the bifurcation phenomena on vehicle spin and system instability were examined using the Perturbation method. The joint-point locus approach is introduced in [18] to geometrically analyze the equilibria of the system and their associated stability properties. The authors have shown that a vehicle can remain stable even when pushed to its limits. Although the attractive domain of stable poles is very narrow in the presented conditions, stability in highly nonlinear regions can still be maintained. Wang et al. has presented a new perspective to the stability analysis of ground vehicles in [19] by taking the driving model into account. Consistent with the fact that at high speeds there is a significant difference between FWD and RWD modes, the authors have shown that the existing stability-estimation techniques will fail because they fail to consider the drive model. Della Rossa et al. [20] has explored a special condition, where stability is a concern when vehicles are negotiating a curve. A 2DOF model with nonlinear tires is chosen to study the steady-state cornering condition. A set of Homoclinic bifurcations, stable, and unstable limit cycles are identified when the the vehicle, is exposed to the conditions reported by professional driver as rare but very dangerous. In all of the aforementioned studies, the pure-slip tire model is employed to avoid the complexities in analytic or even numerical methods. However, in the extreme driving conditions and nonlinear operating regions investigated by most articles, the combined-slip (combinedfriction) case is a dominant phenomenon. This characteristic of tire models is considered in this current article to check the slip ratio's adverse effect on the lateral tire capacity.

This paper employs the LuGre tire model [8] and provides a new representation of the tire-vehicle interaction in canonical form, which includes linear and nonlinear parts. The lateral dynamics of a vehicle with conventional linear tires is provided in Section II. Section III recalls the pure/combinedslip LuGre tire models. Using the steady state and transient LuGre models, section IV explores the lateral dynamics arising from the general form of the pure/combined-slip approaches. The performance and robustness of the developed tire-vehicle interaction are examined and compared with those of the conventional 2DOF model in Section $\mathrm{V}$. The stability of the developed linear parameter-varying (LPV) tire-vehicle lateral dynamics is analyzed in Section VI. The affine quadratic stability of the proposed LPV 
system is proven and compared to the tight quadratic stability condition. Consequently, speed limit criteria is introduced in accordance to the stability of the linear part of the lateral model. Section VII briefly discusses the application of the dynamics developed in this paper to the controller design. Section VIII compares the performance of the steadystate and transient models before moving on to discuss then discusses the findings on various maneuvers carried out under lateral or longitudinal excitation. The last section includes conclusions and future work.

\section{VEHICLE LATERAL DYNAMICS}

The 2DOF bicycle model, a well-known vehicle lateral model, provides vehicle lateral velocity and yaw rate based on longitudinal and lateral tire forces, $F_{x}$ and $F_{y}$. The lateral dynamic is in the following form with the vehicle mass $m$ and moment of inertia $I_{z}$ :

$$
\begin{gathered}
m(\dot{v}+r u)=F_{x f} \sin \delta+F_{y f} \cos \delta+F_{y r} \\
I_{z} \dot{r}=a\left(F_{x f} \sin \delta+F_{y f} \cos \delta\right)-b F_{y r}
\end{gathered}
$$

where $u$ is the speed, subscripts $f, r$ symbolize front and rear tracks, $a, b$ represent the distance of the front and rear tracks from $\mathrm{CG}, \delta$ is the steering angle on the front wheels, and $v, r$ denote the lateral velocity and the yaw rate respectively. A linear tire model suggests lateral forces at each track $F_{y f}=$ $C_{\alpha_{f}} \alpha_{f}, F_{y r}=C_{\alpha_{r}} \alpha_{r}$, in which $\alpha_{f}, \alpha_{r}$ are the front and rear slip angles, respectively, and $C_{\alpha_{f}}, C_{\alpha_{r}}$ are the cornering stiffness. Front and rear slip angles are related to the lateral states $v, r$ as $\alpha_{f}=\delta-\frac{v+a r}{u}, \alpha_{r}=\frac{r b-v}{u}$. The steering angle effect on the longitudinal/lateral forces can be ignored, and the lateral dynamic equations can be simplified as shown in [21]:

$$
\begin{array}{r}
m(\dot{v}+r u)=F_{y f}+F_{y r} \\
I_{z} \dot{r}=a F_{y f}-b F_{y r} .
\end{array}
$$

Consequently, the linear tire-vehicle handling model can be represented by $\dot{x}=A x+B \delta$, where $B=\left[\begin{array}{ll}\frac{C_{\alpha_{f}}}{m} & a \frac{C_{\alpha_{f}}}{I_{z}}\end{array}\right]^{T}$ and

$$
A=\left[\begin{array}{cc}
-\frac{C_{\alpha_{f}}+C_{\alpha_{r}}}{u m} & -\left(\frac{a C_{\alpha_{f}}-b C_{\alpha_{r}}}{u m}+u\right) \\
-\frac{a C_{\alpha_{f}}-b C_{\alpha_{r}}}{u I_{z}} & -\frac{a^{2} C_{\alpha_{f}}+b^{2} C_{\alpha_{r}}}{u I_{z}}
\end{array}\right],
$$

in which the vehicle states are defined as $x=\left[\begin{array}{ll}v(t) & r(t)\end{array}\right]^{T}$. Under the constant velocity assumption, the stability of the above time invariant system suggests the condition

$$
l+K_{u s} u^{2}>0,
$$

with the understeer coefficient $K_{u s}=\frac{-m\left(a C_{\alpha_{f}}-b C_{\alpha_{r}}\right)}{l C_{\alpha_{f}} C_{\alpha_{r}}}$ and $l=a+b$. As pointed out above, the bicycle model is obtained under the assumption that the relation between lateral force and slip angle is linear. However, this assumption is not always realistic. More specifically, the relation between the kinematic variables (like slip ratio or slip angle) and the forces of the tire is nonlinear in general. Moreover (3) is independent of the slip ratio; i.e., the value of the longitudinal slip ratio has not been considered there. In this paper, the force components $F_{y f}$ and $F_{y r}$ are replaced with the LuGre tire model, which captures the nonlinearity of the force-slip relation. The effect of the pure and combined-slip models has also been incorporated in this study, and the developed lateral dynamics seems more practical to be used for the sideslip angle estimator or the stability control. Consequently, the derived lateral dynamics will lead to some specific characterization and conditions for stability which can be compared with those in (4). Two vehicles, an SUV and a sedan, are used in this paper as examples to verify the lateral responses. Specifications of these vehicle are listed in Table I.

\section{LuGRE TIRE MODEL}

This section formulates the averaged lumped LuGre friction model, which can be implemented in the lateral dynamics.

Numerous studies on vehicle state estimation [22]-[25] or stability analysis [15] have documented tire-road forces even in the nonlinear region, represented by a group of curves, among which, the most commonly used are those of algebraic force-slip relationships such as Pacejka model [2]. Compared to other conventional approaches, e.g. Pacejka, the LuGre model utilizes relative velocities $v_{r x}=R_{e} \omega-u$ and $v_{r y}=u \alpha$ rather than slip ratio $\lambda=\frac{v_{r x}}{\max \left\{R_{e} \omega, u\right\}}$ and slip angle $\alpha$ where $\omega$ is the wheel speed and $R_{e}$ is the tire's effective radius. The passivity of the transient LuGre makes it a bounded and stable model and prohibits the divergence of both internal tire states and consequent forces [26]. Accurate force results will be obtained by considering normal force distributions over the contact patch and multiple bristle contact points. The average lumped LuGre model [27] symbolizes the distributed force over the patch line with some simplifications of normal force distribution; representing average deflection of the bristles, the tire internal lateral state $\bar{z}_{y}$ in the average lumped LuGre model relates the relative lateral velocity $v_{r y}=u \alpha$ and tire parameters as:

$$
\dot{\bar{z}}_{y}(\zeta, t)=v_{r y}-\left(\frac{\sigma_{0}\left|v_{r y}\right|}{\theta g\left(v_{r y}\right)}+\kappa R_{e}|\omega|\right) \bar{z}_{y}(\zeta, t),
$$

in which $\zeta$ is the the axis coordinate. The rubber stiffness $\sigma_{0}$ and road friction condition $\theta$ are defined in Table I. The force distribution along the patch line is represented by parameter $\kappa$ in the average lumped model and can be a function of time, a constant, or may be approximated by an asymmetric trapezoidal scheme. The suggested value for $\kappa$ in [27] is $\kappa=\frac{7}{6 L}$, where $L$ is the tire patch length. Moreover, $g\left(v_{r y}\right)$ is defined by:

$$
g\left(v_{r y}\right)=\mu_{c}+\left(\mu_{s}-\mu_{c}\right) e^{-\left|\frac{v_{r y}}{V_{s}}\right|^{\bar{\tau}}},
$$

where the tire parameter $\bar{\tau}=0.5$ is assumed for the distributed LuGre model [27] and $\mu_{c}, \mu_{s}$ are the normalized Coulomb and static friction, respectively. The Stribeck velocity $V_{s}$ shows the transition between these two friction states. In the current study, identification of the LuGre tire parameters was done using the experimental curves of the Chevrolet Equinox standard tires and by utilizing an error cost function and the Nonlinear Least Square method. The 
tire curve resulting from the parameters identified in the lateral direction is compared with the experimental tire curve in Fig. .11 in the Appendix. The parameters listed in Table I [28] will be used in this study, and they were obtained using the nonlinear least square identification method.

TABLE I: Vehicle Spec. \& LuGre Model Parameters

\begin{tabular}{|c|c|c|c|c|}
\hline Parameter & Unit & Value & & Description \\
\hline$\sigma_{0}$ & {$[1 / m]$} & 181.5 & & Rubber stiffness \\
\hline$\sigma_{1}$ & {$[s / m]$} & 0.9 & & Rubber damping \\
\hline$\sigma_{2}$ & {$[s / m]$} & 0.001 & & Relative viscous damping \\
\hline$\mu_{s}$ & {$[-]$} & 1.55 & & Normalized static frict. \\
\hline$\mu_{c}$ & {$[-]$} & 0.85 & & Front \& rear axles to $\mathrm{CG}$ \\
\hline$V_{s}$ & {$[\mathrm{~m} / \mathrm{s}]$} & 6.6 & & Stribeck relative velocity \\
\hline$\kappa$ & {$[-]$} & 8.3 & & Load distribution factor \\
\hline Parameter & Unit & SUV & Sedan & Description \\
\hline$m$ & {$[k g]$} & 2270 & 1530 & Vehicle mass \\
\hline$I_{z}$ & {$\left[k g m^{2}\right]$} & 4600 & 4192 & Moment of inertia \\
\hline$a$ & {$[\mathrm{~mm}]$} & 1421 & 1320 & Front axle to $\mathrm{CG}$ \\
\hline$b$ & {$[\mathrm{~mm}]$} & 1438 & 1456 & Rear axle to $\mathrm{CG}$ \\
\hline$C_{\alpha_{f}}$ & {$[N / \mathrm{rad}]$} & 69800 & 70000 & Front cornering stiffness \\
\hline$C_{\alpha_{r}}$ & {$[N / \mathrm{rad}]$} & 69600 & 69900 & Rear cornering stiffness \\
\hline
\end{tabular}

Equations (5), (6) are developed based on the pure-slip condition, which cannot address the issue of decreasing lateral tire capacities due to the longitudinal slip. The combined-slip (direct correlation between the lateral and longitudinal slips) LuGre model is proposed by Velenis [9], in which the internal state $\bar{z}_{j}, j \in\{x, y\}$ for each direction is described as:

$$
\dot{\bar{z}}_{j}=v_{r j}-C_{0 j} \bar{z}_{j}-\kappa R_{e}|\omega| \bar{z}_{j},
$$

where $C_{0 j}=\frac{\left\|M_{c}^{2} \mathbf{v}_{\mathbf{r}}\right\| \sigma_{0 j}}{g\left(\mathbf{v}_{\mathbf{r}}\right) \mu_{c j}^{2}}$, and $M_{c}=\left[\begin{array}{lll}\mu_{c x} & 0 ; 0 & \mu_{c y}\end{array}\right]$. The transient function $g\left(\mathbf{v}_{\mathbf{r}}\right)$ between the Columb and static friction in the combined slip tire model is introduced in [29] as:

$$
g\left(\mathbf{v}_{\mathbf{r}}\right)=\frac{\left\|M_{c}^{2} \mathbf{v}_{\mathbf{r}}\right\|}{\left\|M_{c} \mathbf{v}_{\mathbf{r}}\right\|}+\left(\frac{\left\|M_{s}^{2} \mathbf{v}_{\mathbf{r}}\right\|}{\left\|M_{s} \mathbf{v}_{\mathbf{r}}\right\|}-\frac{\left\|M_{c}^{2} \mathbf{v}_{\mathbf{r}}\right\|}{\left\|M_{c} \mathbf{v}_{\mathbf{r}}\right\|}\right) e^{-\left|\frac{\left\|\mathbf{v}_{\mathbf{r}}\right\|}{V_{s}}\right|^{0.5}},
$$

where $M_{s}=\left[\begin{array}{lll}\mu_{s x} & 0 ; 0 & \mu_{s y}\end{array}\right]$ and $\mathbf{v}_{\mathbf{r}}=\left[\begin{array}{ll}v_{r x} & v_{r y}\end{array}\right]^{T}$. The final form of the normalized friction force $\left(\mu_{j}=\frac{F_{j}}{F_{z j}}\right)$ of the averaged lumped LuGre model with $\overline{\mathbf{z}}=\left[\begin{array}{ll}\bar{z}_{y} & \bar{z}_{x}\end{array}\right]^{T}$ yields [29]

$$
\mu=\sigma_{0} \overline{\mathbf{z}}+\sigma_{1} \dot{\overline{\mathbf{z}}}+\sigma_{2} \mathbf{v}_{r},
$$

in which $\mu, \overline{\mathbf{z}}, \mathbf{v}_{r} \in \mathbb{R}^{2}$ and can be described both in longitudinal and lateral directions in the combined or unidirectional slip models. The longitudinal relative velocity is defined by $v_{r x}=\lambda R_{e} \omega$ and $v_{r x}=\lambda u$ for the traction and brake cases, respectively. In addition, the rubber stiffness is $\sigma_{0}=\left[\begin{array}{lll}\sigma_{0 x} & 0 ; 0 & \sigma_{0 y}\end{array}\right]$, the rubber damping is $\sigma_{1}=$ $\left[\begin{array}{lll}\sigma_{1 x} & 0 ; 0 & \sigma_{1 y}\end{array}\right]$, and the relative viscous damping is defined by $\sigma_{2}=\left[\begin{array}{lll}\sigma_{2 x} & 0 ; 0 & \sigma_{2 y}\end{array}\right]$, in which $\sigma_{0 j}, \sigma_{1 j}$ and $\sigma_{2 j}$ are the rubber stiffness, damping, and relative viscous damping in each direction. These pure and combined-slip models can be used in road-independent state estimation approaches
[30], will be incorporated in the lateral dynamics, and are described in the next section.

\section{LAteral Dynamics With the LuGre Tire Model}

In Section II, a linear tire model is assumed and used to derive the lateral dynamic equation (3). However, this assumption is not realistic due to the nonlinear nature of tire-road interactions. After discussing the LuGre model for both pure and combined-slip cases in Section III, we incorporate it into the vehicle lateral dynamics (2) in this section. This analysis leads to a formulation that captures nonlinearities in the model as well as the pure and combinedslip characteristics. The pure-slip model is addressed first. It contains the steady state and transient assumptions. Then, the combined friction case is investigated and the closed form of the combined-slip with lateral dynamics is provided to facilitate the stability analysis. The suggested formulations are also useful for the control and estimation applications.

\section{A. Lateral Dynamics with the Pure-Slip Condition}

In this subsection both the steady-state and transient forms of the pure-slip LuGre model (5) are incorporated into the lateral dynamics (2), and formulae, are derived for the linear part of the curves and the saturation/nonlinear regions. Comparison of the steady-state assumption of the LuGre model with (5) is left to the end of this section.

1) Steady-state LuGre model: If we assume the steady state LuGre model $\left(\dot{\bar{z}}_{y}(\zeta, t)=0\right)$ by defining new variables

$$
\rho=\theta g\left(v_{r y}\right), \quad \gamma=\kappa R_{e} \omega / \sigma_{0 y},
$$

the normalized lateral force $\mu_{y}$ will be as follows with $\alpha$ as the slip angle at each tire/axle. (Detail of this derivation is provided in the Appendix)

$$
\mu_{y}=\left(\frac{\rho}{u|\alpha|+\gamma \rho}+\sigma_{2 y}\right) u \alpha .
$$

To be able to write the state-space form of the lateral dynamics based on the LuGre model, we need to analyze the effect of the slip angle as discussed in the following sections. For the case where $|\alpha| \ll \gamma \rho / u$, the normalized lateral force (11) will be

$$
\mu_{y}=\theta\left(\frac{1}{\gamma}+\sigma_{2 y}\right) u \alpha
$$

Here, $\theta$ is employed with direct multiplication as an implication of the effect of road conditions. It helps in making the suggested pure-slip formulation compatible with the real tire model since the slope of the linear region of the forceslip curve is a function of the road condition as studied in the slip-slope method [31], [32]. Normalized lateral forces of the pure-slip LuGre model and the linear part (that resembles $|\alpha| \ll \gamma \rho / u$ ) are depicted in Fig. 1 for various road conditions.

The lateral dynamics with the tire model can be expressed as follows after putting the tire forces of each track $F_{y i}=$ $\mu_{y i} F_{z i}$ in (2)

$$
\begin{array}{r}
m(\dot{v}+r u)=\mu_{y f} F_{z f}+\mu_{y r} F_{z r} \\
I_{z} \dot{r}=a \mu_{y f} F_{z f}-b \mu_{y r} F_{z r},
\end{array}
$$




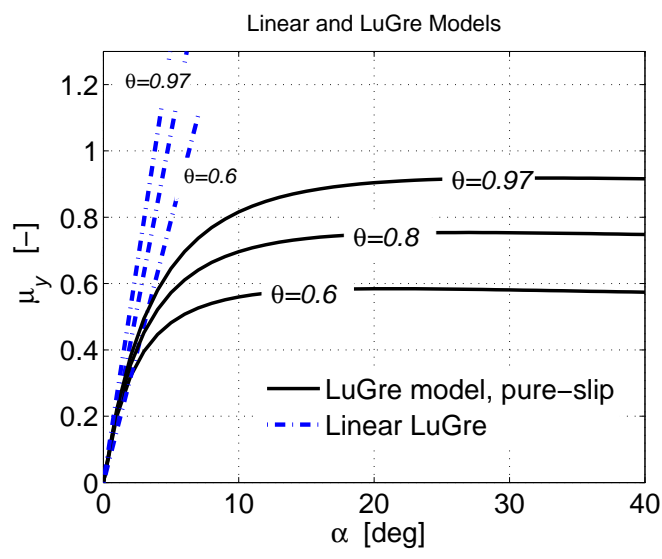

Fig. 1: Pure-slip LuGre lateral tire model

Several studies focuses on normal force calculation on each axle using load transfer and acceleration measurements [28], [33], [34]. Calculated normal forces on the front and rear axles $F_{z f}$ and $F_{z r}$ can then be utilized in (13) whenever lateral/longitudinal acceleration measurements are available. To reach a stability condition comparable with the conventional understeer coefficient (4), a static normal load distribution is used in this subsection, which results in the following vehicle state form after substituting the corresponding lateral forces from (12)

$\dot{v}=-\frac{g}{l}\left(b k_{f}+a k_{r}\right) v-\left(\frac{a b g}{l}\left(k_{f}-k_{r}\right)+u\right) r+\frac{b g}{l} k_{f} u \delta$, $\dot{r}=\frac{m a b g}{I_{z} l}\left(k_{r}-k_{f}\right) v-\frac{m a b g}{I_{z} l}\left(b k_{r}+a k_{f}\right) r+\frac{m a b g}{I_{z} l} k_{f} u \delta$,

where $k_{f}=\theta\left(\frac{1}{\gamma_{f}}+\sigma_{2 f}\right)$ and $k_{r}=\theta\left(\frac{1}{\gamma_{r}}+\sigma_{2 r}\right)$. Equation (14) represents a linear time-varying system for the lateral dynamics with states $x=\left[\begin{array}{ll}v(t) & r(t)\end{array}\right]^{T}$ and front steering; it is based on the LuGre linearized model for small slip angles. To consider the nonlinear part, disregarding the $|\alpha| \ll \gamma \rho / u$ condition, one can rewrite the normalized lateral force (11) at each corner

$$
\mu_{y i}=k_{i} u \alpha_{i}-\frac{1}{\gamma_{i}\left(1+\frac{\gamma_{i} \rho_{i}}{u\left|\alpha_{i}\right|}\right)} u \alpha_{i}
$$

where $i \in\{f, r\}$ can be front or rear tires, $\rho_{i}, \gamma_{i}$ are defined in (10), $u$ is the vehicle speed, and $\alpha_{i}$ is the slip angle at each track. The term $k_{i} u \alpha$ represents the linear part (12), and the second term shows nonlinear behavior of the lateral force with respect to the slip angle. By substituting (15) in the lateral dynamics (13), one will get ${ }^{1}$ :

$$
\dot{x}=A(t) x+B(t) \delta_{f}+H(t)
$$

\footnotetext{
${ }^{1}$ From now on, since most of the matrices (state matrices or nonlinear parts) contain time varying parameters $\omega(t)$, the notations $\mathcal{M}, \mathcal{M}(t)$ or $\mathcal{M}(\omega)$ may be used for any matrix like $\mathcal{M}($.$) interchangeably.$
}

where $B(t)=\left[\begin{array}{ll}\frac{b g}{l} k_{f} u & \frac{m a b g}{I_{z} l} k_{f} u\end{array}\right]^{T}$ and $A(t)$ is defined by

$$
A(t)=\left[\begin{array}{cc}
\frac{-g}{l}\left(b k_{f}+a k_{r}\right) & -\left(\frac{a b g}{l}\left(k_{f}-k_{r}\right)+u\right) \\
\frac{m a b g}{I_{z} l}\left(k_{r}-k_{f}\right) & -\frac{m a b g}{I_{z} l}\left(b k_{r}+a k_{f}\right)
\end{array}\right] .
$$

The components of $H(t)$ representing the nonlinear region are

$$
H(t)=\left[\begin{array}{c}
\frac{g u}{l}\left(b \phi_{f} \alpha_{f}+a \phi_{r} \alpha_{r}\right) \\
\frac{m a b g u}{I_{z} l}\left(\phi_{f} \alpha_{f}-\phi_{r} \alpha_{r}\right)
\end{array}\right],
$$

in which $\phi_{f}$ and $\phi_{r}$ are obtained as follows for the front and rear tires, respectively

$$
\phi_{i}=\left(\frac{\rho_{i}}{u\left|\alpha_{i}\right|+\gamma_{i} \rho_{i}}-\frac{\theta}{\gamma_{i}}\right) .
$$

The linear part, $A(t)$, of system (17) is parameter-varying due to the varying wheel speed $\omega(t)$ in $\gamma_{f}, \gamma_{r}$ and in $k_{f}, k_{r}$ consequently. The stability of this system will be investigated in Section V.

Remark 1: The linear part of the state-space (16) for the pure-slip condition can be directly compared with its traditional counterpart (3).

Previously, we assumed that $|\alpha| \ll \gamma \rho / u$, which covers the linear region from the spectrum of the behaviour of $\alpha$. Now, we discuss the convenience of the assumption $|\alpha| \ll$ $\gamma \rho / u$ for linearized lateral dynamics. In Fig. 2, we show the effect of the slip angle $\alpha$ on $\|H(t)\|$, the Euclidean norm of the nonlinear part (18) on the SUV with the specifications summarized in Table I.

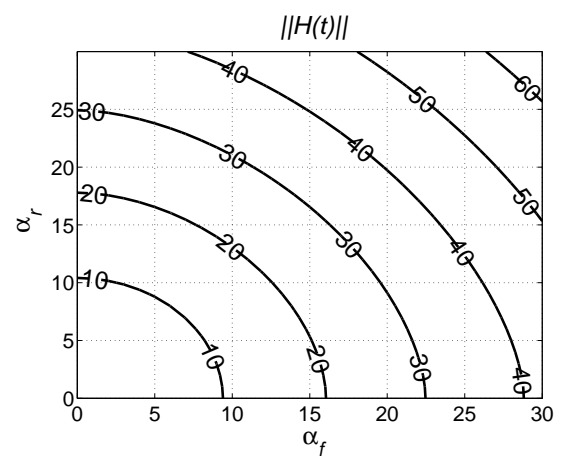

Fig. 2: Effect of slip angle on $\|H(t)\|$ for the pure-slip model on dry, $u=54[k p h]$

Figure 2 depicts the magnitude of the nonlinear term $H(t)$, which is a bounded function of the front and rear slip angles. Equation (16) can be utilized as a control system and an appropriate controller to mitigate the effect of this nonlinear term as discussed in Section VII.

Remark 2: Equation (16) can be rewritten using normal forces on each axle $F_{z f}$ and $F_{z r}$ instead of utilizing the static normal load distribution. This leads to the following lateral dynamics:

$$
\dot{x}=A_{n}(t) x+B_{n}(t) \delta_{f}+H_{n}(t),
$$


where $B_{n}(t)=\left[\begin{array}{ll}\frac{F_{z f}}{m} k_{f} u & \frac{a F_{z f}}{I_{z}} k_{f} u\end{array}\right]^{T}$ and $A_{n}(t)$ is defined by

$$
A_{n}(t)=\left[\begin{array}{cc}
-\left(\frac{F_{z f}}{m} k_{f}+\frac{F_{z r}}{m} k_{r}\right) & \frac{b F_{z r}}{m} k_{r}-\frac{a F_{z f}}{m} k_{f}-u \\
\frac{b F_{z r}}{I_{z}} k_{r}-\frac{a F_{z f}}{I_{z}} k_{f} & -\left(\frac{b^{2} F_{z r}}{I_{z}} k_{r}+\frac{a^{2} F_{z f}}{I_{z}} k_{f}\right)
\end{array}\right]
$$

The nonlinear part also changes to

$$
H_{n}(t)=\left[\begin{array}{c}
\frac{u}{m}\left(F_{z f} \phi_{f} \alpha_{f}+F_{z r} \phi_{r} \alpha_{r}\right) \\
\frac{u}{I_{z}}\left(a F_{z f} \phi_{f} \alpha_{f}-b F_{z r} \phi_{r} \alpha_{r}\right)
\end{array}\right]
$$

Remark 3: General form of the pure-slip vehicle-tire lateral dynamics can be rewritten in a linear time-varying system for large slip angle. In the case in which the slip angles satisfy $|\alpha| \gg \gamma \rho / u$ (large slip angles), the normalized lateral force (11) will be $\mu_{y}=\rho \operatorname{sgn}(\alpha)+\sigma_{2 y} u \alpha$. Thus, the nonlinear part $H(t)$ can be written in a form that contains states $v$ and $r$. This is done by the assumption of $|\alpha| \gg$ $\gamma \rho / u$, which simplifies the term $1+\gamma \rho /(u \alpha)$ to 1 . Thus, at very high slip angles $|\alpha| \gg \gamma \rho / u$, the term $H(t)$ in (16) can be expressed as:

$$
H(t)=\mathscr{A}(t) x+\mathscr{B}(t) \delta
$$

where $\mathscr{B}(t)=\frac{-\theta b g u}{l}\left[\begin{array}{ll}\frac{1}{\gamma_{f}} & \frac{m a}{I_{z}}\end{array}\right]^{T}$ and $\mathscr{A}(t)$ is

$$
\mathscr{A}(t)=\left[\begin{array}{cc}
-A(1,1)-q_{1} & -A(1,2)-q_{2}-u \\
-A(2,1)-q_{3} & -A(2,2)-q_{4}
\end{array}\right]
$$

where $A(i, j)$ is the $(i, j)$-th element of $A$ in (17) and $q$ 's are:

$$
\begin{aligned}
& q_{1}=\frac{\theta g}{l}\left(b \sigma_{2 f}+a \sigma_{2 r}\right) ; q_{2}=\frac{\theta a b g}{l}\left(\sigma_{2 f}-\sigma_{2 r}\right) \\
& q_{3}=\frac{\theta m a b g}{I_{z} l}\left(\sigma_{2 f}-\sigma_{2 r}\right) ; q_{4}=\frac{\theta m a b g}{I_{z} l}\left(a \sigma_{2 f}+b \sigma_{2 r}\right)
\end{aligned}
$$

Therefore, for large slip angles, the lateral dynamics has the following linear form

$$
\dot{x}(t)=(A+\mathscr{A}) x(t)+(B+\mathscr{B}) \delta
$$

2) Transient LuGre model: In this part, the state space form of the transient pure-slip case is derived and it is concluded that the steady-state case, which will be employed in this study, provides reasonable accuracy. The average lumped LuGre model (5) for the pure-slip condition and $\omega \geq 0$ can be expressed as $\dot{\bar{z}}_{y}=-\mathcal{E}(t) \bar{z}_{y}+v_{r y}$ with

$$
\mathcal{E}(t)=\frac{\sigma_{0} u}{\rho}\left(\frac{\gamma \rho}{u}+|\alpha|\right)
$$

and $\gamma, \rho$ defined in (10). The solution of (5) is

$$
\bar{z}_{y}=\bar{z}_{y}(0) e^{-t \mathcal{E}(t)}+\int_{0}^{t} e^{-\mathcal{E}(\tau)(t-\tau)} v_{r y} d \tau .
$$

The condition $|\alpha| \ll \gamma \rho / u$ for the linear part leads to $\mathcal{E}(t)=\sigma_{0} \gamma=\kappa R_{e} \omega(t)$ which simplifies (28) to $\bar{z}_{y}=$ $\int_{0}^{t} e^{-\kappa R_{e} \omega(\tau)(t-\tau)} u \alpha d \tau$, assuming zero initial condition.
Considering $\omega(t) \in\left[\omega_{l}, \omega_{u}\right]$ for all $t \geq 0$ where $\omega_{l}, \omega_{u}$ are the lower and upper bounds of the wheel speed together with the fact that the above integral is an increasing function of $\tau$ we have $\frac{u \alpha}{\kappa R \omega_{u}}\left(1-e^{-\kappa R \omega_{u} t}\right) \leq \bar{z}_{y} \leq \frac{u \alpha}{\kappa R \omega_{l}}\left(1-e^{-\kappa R \omega_{l} t}\right)$. Thus, the linear part of the normalized lateral force can be written as follows

$$
\begin{aligned}
\mu_{y i} & =\theta\left[\frac{1}{\gamma_{i}}+e^{-\kappa_{i} R_{e} \omega t}\left(\sigma_{1 i}-\frac{1}{\gamma_{i}}\right)+\sigma_{2 i}\right] u \alpha_{i} \\
& =\bar{k}_{i} u \alpha_{i}
\end{aligned}
$$

with $i \in\{f, r\}$. By applying (29) in (13), the lateral dynamics based on the pure-slip transient LuGre model yields

$$
\dot{x}=\bar{A} x+\bar{B} u+\bar{H},
$$

in which $\bar{A}$ is the same as $A$ in (17), but $k_{f}, k_{r}$ should be replaced with $\bar{k}_{f}, \bar{k}_{r}$ respectively. The time-varying coefficients $\bar{k}_{f}, \bar{k}_{r}$ are defined in (29) for the front and rear tracks. Details of the calculation are provided in the Appendix. The nonlinear part $\bar{H}$ is the same as in (18), but $\phi_{f}, \phi_{r}$ should be replaced with $\bar{\phi}_{f}, \bar{\phi}_{r}$, respectively, where $\bar{\phi}_{i}=\phi_{i}+\Phi_{i}$ and

$$
\begin{aligned}
\Phi_{i} & =e^{-\sigma_{0 i} \gamma_{i} t}\left[\left(\sigma_{1 i}-\frac{\rho_{i}}{u\left|\alpha_{i}\right|+\gamma_{i} \rho_{i}}\right) e^{\frac{-\sigma_{0 i} u\left|\alpha_{i}\right| t}{\rho_{i}}}\right] \\
& -e^{-\sigma_{0 i} \gamma_{i} t} \theta\left(\sigma_{1 i}-\frac{1}{\gamma_{i}}\right)
\end{aligned}
$$

Therefore, the transient model converges very quickly to the steady-state model. In (30) and (29), as time goes on, the exponential terms will tend to zero. Thus, $\Phi_{i} \rightarrow 0$ and (29) tends to (12).

The yaw rate response of the pure-slip steady state and transient models (16) and (29) (with linear and nonlinear terms) are depicted in Fig. 3 and compared with the linear tire-vehicle model (3). The tested vehicle is the SUV provided in Table I, and the test is a step steer on a dry road with longitudinal velocity $u=65[\mathrm{kph}]$, and steering angle $\delta=0.035[\mathrm{rad}]$ on the front wheels. The transient and steady state lumped LuGre models respond similarly to this input.

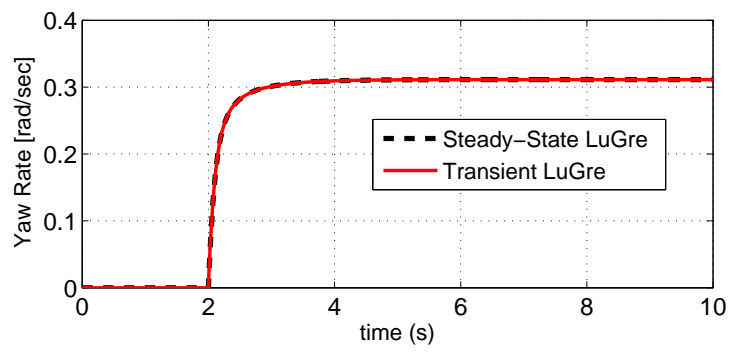

Fig. 3: Step steer, steady-state vs. transient LuGre model

The difference between the LuGre and the linear tire occur because of the existing nonlinear terms in the LuGre, which correctly represent the lateral tire forces and consequently provide a more accurate yaw rate and lateral velocity responses. The cornering stiffnesses for each front and rear tire in the lateral dynamic model (3) with linear 
tires are considered, as $C_{\alpha f}=69800[N / \mathrm{rad}]$ and $C_{\alpha r}=$ $69600[\mathrm{~N} / \mathrm{rad}]$ respectively. These values are compatible in the linear region with the LuGre parameters provided in Table I. More results of the suggested steady state (16) and transient models (29) and (30) are provided in Section VI, specifically in Table II, and confirms the accuracy of the steady-state approach.

\section{B. Combined-Slip LuGre Model in Lateral Dynamics}

The studies in the previous subsection and simulations in Section VI show that the steady-state model provides reasonable accuracy for several lateral tests, which makes it a sound choice for implementation in the derivation of the lateral dynamics with combined-slip friction and tire nonlinearities. Subsequently, the tire-vehicle lateral dynamics with the steady-state combined-slip LuGre model is developed in this part. A practical, closed form lateral vehicle-tire model that includes combined friction characteristics and a consideration of a slip ratio on each wheel is presented. This is an advantage of the current formulation over conventional lateral vehicle-tire approaches that assume pure-slip and work with each track instead of each corner. A general vehicle model, with two conventional degrees of freedom $v, r$ along with the longitudinal slip ratio $\lambda$ at each corner, is utilized in this section and shown in Fig. 4.

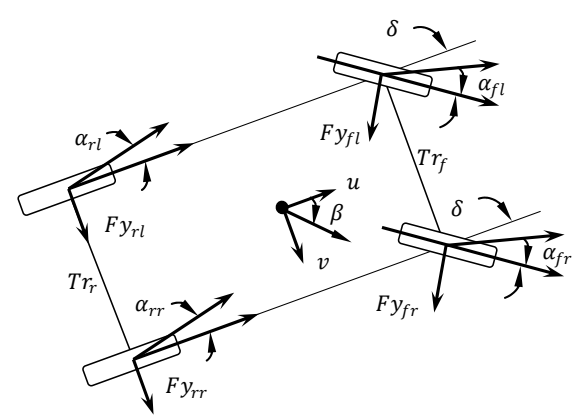

Fig. 4: Planar vehicle model for the combined-slip analysis

The combined-slip scheme incorporates the effect of the slip ratio, $\lambda$, and slip angle, $\alpha$, simultaneously at each corner, which provides a more practical tire model. The steady-state combined-slip LuGre model with $\omega>0$ yields

$$
z_{y}=\frac{v_{r y}}{\kappa R_{e} \omega+\frac{\left\|M_{c}^{2} \mathbf{v}_{\mathbf{r}}\right\| \sigma_{0 y}}{\theta g\left(\mathbf{v}_{\mathbf{r}}\right) \mu_{c y}^{2}}} .
$$

Putting the longitudinal relative velocity $v_{r x}=R_{e} \omega-u$ and lateral relative velocity $v_{r y}=u \alpha$ in (31) and using (9), one can obtain the following normalized lateral force of the combined-slip LuGre model:

$$
\mu_{y}=\left(\frac{\sigma_{0 y}}{\kappa R_{e} \omega+\frac{\sigma_{0 y} \sqrt{\psi}}{\theta g\left(\mathbf{v}_{\mathbf{r}}\right)}}+\sigma_{2 y}\right) u \alpha
$$

where $\psi=u^{2} \alpha^{2}\left[1+\left(\eta \lambda R_{e} \omega / u \alpha\right)^{2}\right]$ and $\eta=\left(\mu_{c x} / \mu_{c y}\right)^{2}$.
1) Deviation from the pure slip condition: One can define a metric to measure how far the system is from the pure slip condition. This metric is in terms of the ratio between the slip ratio and slip angle. More specifically, based on (32), we introduce $\lambda R_{e} \omega / u \alpha$ as a metric that can be used to identify this distance. For pure slip, i.e., $\lambda R_{e} \omega / u \alpha \ll 1$, equation (32) changes to (11), which was investigated in the previous subsection. On the other hand, when $\lambda R_{e} \omega / u \alpha \gg 1$, (32) can be written as

$$
\mu_{y}=\left(\frac{\rho}{\epsilon|\lambda|+\gamma \rho}+\sigma_{2 y}\right) u \alpha=\mathcal{K} u \alpha,
$$

in which $\epsilon=\eta R_{e} \omega$. This represents a linear force-slip angle relationship for the large slip ratios, as illustrated in Fig. 5 by dashed lines. Equation (33) also substantiates the linear

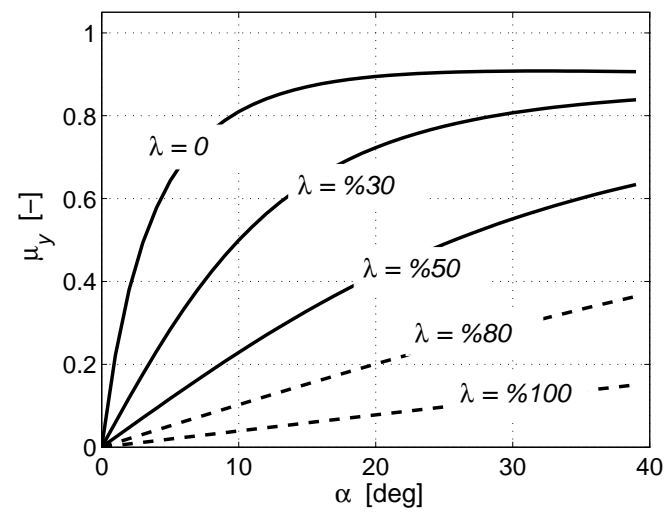

Fig. 5: Normalized lateral forces, combined-slip model, on a dry $\operatorname{road} \theta=1$ with longitudinal speed $u=20[\mathrm{~m} / \mathrm{s}]$.

characteristics of all combined-slip tire curves for small slip angles $\alpha_{i}$ as shown in Fig. 5.

2) General form of the combined-slip model: The general form of the lateral LuGre tire forces in (32) with the combined-slip condition, can be rewritten as

$$
\mu_{y}=\left(\frac{\rho}{\sqrt{u^{2} \alpha^{2}+\lambda^{2} \epsilon^{2}}+\gamma \rho}+\sigma_{2 y}\right) u \alpha
$$

Since this is a combined-slip model, the wheel speed and slip ratio at each corner (wheel) make a significant contribution in the lateral forces and the lateral dynamics. This contribution is considered when implementing both the linear part and general form (34) of the lateral force and static normal force, $F_{z i}$, distribution at each corner, which results in

$$
\dot{x}=A^{\prime}(t) x+B^{\prime}(t) \delta_{f}+L(t),
$$

where $B^{\prime}(t)=\left[\begin{array}{ll}\frac{b g}{l} \mathcal{P}_{f} u & \frac{m a b g}{I_{z} l} \mathcal{P}_{f} u\end{array}\right]^{T}$, and $A^{\prime}(t)$ is

$$
A^{\prime}(t)=\left[\begin{array}{cc}
\frac{-g}{2 l}\left(b \mathcal{P}_{f}+a \mathcal{P}_{r}\right) & -\left(\frac{a b g}{2 l}\left(\mathcal{P}_{f}-\mathcal{P}_{r}\right)+u\right) \\
\frac{m a b g}{2 I_{z} l}\left(\mathcal{P}_{r}-\mathcal{P}_{f}\right) & -\frac{m a b g}{2 I_{z} l}\left(b \mathcal{P}_{r}+a \mathcal{P}_{f}\right)
\end{array}\right],
$$

where $\mathcal{P}_{f}=k_{f l}^{\prime}+k_{f r}^{\prime}, \mathcal{P}_{r}=k_{r l}^{\prime}+k_{r r}^{\prime}$. The parameters $k_{i}^{\prime} \mathrm{s}$ are defined at each corner $i \in\{f l, f r, r l, r r\}$ independently:

$$
k_{i}^{\prime}=\frac{\sigma_{2 y i}+\rho_{i}}{\epsilon_{i}\left|\lambda_{i}\right|+\gamma_{i} \rho_{i}} .
$$


The nonlinear term $L(t)$ can be written as follows with $\mathcal{Q}_{f}=$ $\mathcal{M}_{f l}+\mathcal{M}_{f r}$ and $\mathcal{Q}_{r}=\mathcal{M}_{r l}+\mathcal{M}_{r r}$ for the front and rear tracks:

$$
L(t)=\left[\begin{array}{c}
\frac{b g u \alpha_{f}}{2 l} \mathcal{Q}_{f}+\frac{a g u \alpha_{r}}{2 l} \mathcal{Q}_{r} \\
\frac{m a b g u}{2 I_{z} l}\left(\alpha_{f} \mathcal{Q}_{f}-\alpha_{r} \mathcal{Q}_{r}\right)
\end{array}\right]
$$

where $\rho_{i}, \gamma_{i}$ are from (10), $N_{i}=\frac{\sqrt{u_{i}^{2} \alpha_{i}^{2}+\epsilon_{i}^{2} \lambda_{i}^{2}}}{\rho_{i}+\gamma_{i}}$, and $\mathcal{M}_{i}=$ $\frac{1}{N_{i}}-k_{i}^{\prime}+\sigma_{2 y i}$ for each tire separately. Fig. 6 represents the effect of the slip angle $\alpha$ on the Euclidean norm of the nonlinear parts (38) on the previously tested SUV.

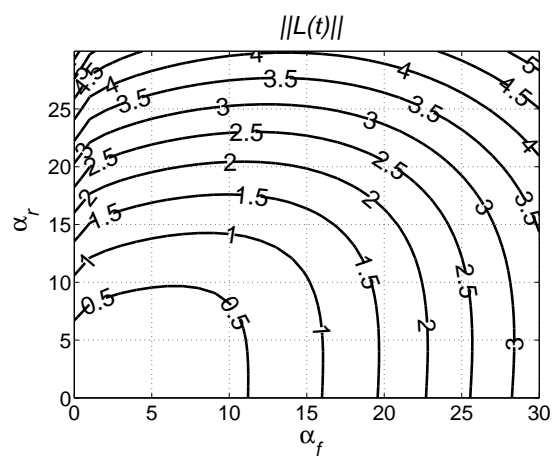

Fig. 6: Effect of slip angles on $\|L(t)\|$, combined-slip model on dry, $u=54[k p h]$ and $\lambda=25 \%$

Remark 4: Considering suspension dynamics and the load transfer, instead of the static normal force distribution, normal forces at each corner $F_{z f l}, F_{z f r}, F_{z r l}, F_{z r r}$ are calculated in [28], [34]. The combined-slip tire-vehicle lateral dynamics (35) can then be rewritten utilizing normal forces as

$$
\dot{x}=A_{n}^{\prime}(t) x+B_{n}^{\prime}(t) \delta_{f}+L_{n}(t),
$$

where $B_{n}^{\prime}(t)=\left[\begin{array}{ll}\frac{u}{m} \mathcal{P}_{f n} & \frac{a u}{I_{z}} \mathcal{P}_{f n}\end{array}\right]^{T}$ and $A_{n}^{\prime}(t)$ is described as follows

$$
A_{n}^{\prime}(t)=\left[\begin{array}{cc}
\frac{-1}{m}\left(\mathcal{P}_{f n}+\mathcal{P}_{r n}\right) & -\left(\frac{a}{m} \mathcal{P}_{f n}-\frac{b}{m} \mathcal{P}_{r n}+u\right) \\
\frac{1}{I_{z}}\left(b \mathcal{P}_{r n}-a \mathcal{P}_{f n}\right) & -\frac{1}{I_{z}}\left(a^{2} \mathcal{P}_{f n}+b^{2} \mathcal{P}_{r n}\right)
\end{array}\right],
$$

in which $\mathcal{P}_{f n}=F_{z f l} k_{f l}^{\prime}+F_{z f r} k_{f r}^{\prime}, \mathcal{P}_{r n}=F_{z r l} k_{r l}^{\prime}+$ $F_{z r r} k_{r r}^{\prime}$. Subsequently, the nonlinear term $L_{n}(t)$ changes to:

$$
L_{n}(t)=\left[\begin{array}{c}
\frac{u \alpha_{f}}{m} \mathcal{Q}_{f n}+\frac{u \alpha_{r}}{m} \mathcal{Q}_{r n} \\
\frac{a u \alpha_{f}}{I_{z}} \mathcal{Q}_{f n}-\frac{b u \alpha_{r}}{I_{z}} \mathcal{Q}_{r n}
\end{array}\right]
$$

where $\mathcal{Q}_{f n}=F_{z f l} \mathcal{M}_{f l}+F_{z f r} \mathcal{M}_{f r}$ and $\mathcal{Q}_{r n}=F_{z r l} \mathcal{M}_{r l}+$ $F_{z r r} \mathcal{M}_{r r}$ for the front and rear axles.

\section{Stability of the Pure And Combined SliP MODELS}

This section provides the speed conditions under which the linear part of the pure-slip and combined-slip models (16), (35) are asymptotically stable. First, the stability with the time-invariant assumption is investigated and speed limit criteria are provided, then quadratic and affine quadratic stability of the parameter-varying case is explored. It is demonstrated that the notion of the affine quadratic stability will give less conservative conditions than the quadratic stability for the lateral dynamics.

\section{A. Time-Invariant Case}

1) Pure-slip model: The stability of the linear part of the system (16) with the pure-slip time-invariant assumption, i.e, constant longitudinal velocity $u$, can be addressed with the Hurwitz criterion on the state matrix $A$ in (17). Having negative eigenvalues yields $m \chi_{2} u^{2}>m \chi_{2}^{2}-\chi_{1} \chi_{3} I_{z}$. Clearly, if $\chi_{2}>0$, (i.e $\frac{\sigma_{0 r}}{\kappa_{r}}>\frac{\sigma_{0 f}}{\kappa_{f}}$ ), there will be no constraint on the longitudinal speed $u$. This resembles the case $K_{u s}>0$ in (4) for the conventional bicycle model with linear tires. However, with the tire parameters provided in Table I, $\chi_{2}<0$ and this results in the following speed limit:

$$
u^{2}<\chi_{2}-\frac{\chi_{1} \chi_{3} I_{z}}{m \chi_{2}}
$$

where $\chi_{1}=\frac{g}{l}\left(\frac{b \sigma_{0 f}}{\kappa_{f}}+\frac{a \sigma_{0 r}}{\kappa_{r}}\right), \chi_{2}=\frac{a b g}{l}\left(\frac{\sigma_{0 r}}{\kappa_{r}}-\frac{\sigma_{0 f}}{\kappa_{f}}\right)$, and $\chi_{3}=\frac{m a b g}{l I_{z}}\left(\frac{a \sigma_{0 f}}{\kappa_{f}}+\frac{b \sigma_{0 r}}{\kappa_{r}}\right)$. This is analogous to the stability condition $u<\sqrt{\frac{-l}{K_{u s}}}$ for the conventional time-invariant case (3) Using the LuGre model parameters provided in Table I, the speed criteria yields $u \leq 63.8[\mathrm{~m} / \mathrm{s}]$, which is close to the $u_{c r}=66[\mathrm{~m} / \mathrm{s}]$ obtained by the condition (4) for the bicycle model, which has tires similar to the linear LuGre model tires.

2) Combined-slip model: The stability of the assumed time-invariant combined-slip state matrix $A^{\prime}$ in (35) can be investigated similar to the pure-slip approach by introducing $s_{i}$ at each corner having the longitudinal slip ratio $\lambda_{i}$ :

$$
s_{i}=\frac{\left(1-\lambda_{i}\right) \rho_{i}}{\eta\left|\lambda_{i}\right|+\frac{\kappa \rho_{i}}{\sigma_{0 i}}}
$$

where $i \in\{f l, f r, r l, r r\}$. Then, the stability condition leads to $m \Gamma_{2} u^{2}>m \Gamma_{2}^{2}-\Gamma_{1} \Gamma_{3} I_{z}$ where:

$$
\begin{aligned}
\Gamma_{1} & =\frac{g}{2 l}\left(b\left(s_{f l}+s_{f r}\right)+a\left(s_{r l}+s_{r r}\right)\right) \\
\Gamma_{2} & =\frac{a b g}{2 l}\left(s_{r l}+s_{r r}-s_{f l}-s_{f r}\right) \\
\Gamma_{3} & =\frac{m a b g}{2 l I_{z}}\left(a\left(s_{f l}+s_{f r}\right)+b\left(s_{r l}+s_{r r}\right)\right)
\end{aligned}
$$

Clearly, if $\Gamma_{2}>0$ (this depends on the slip ratio as well as the tire parameters at each corner), there is no criteria for the longitudinal speed that is unfeasible in practice. Having $\Gamma_{2}<0$ result in:

$$
u^{2}<\Gamma_{2}-\frac{\Gamma_{1} \Gamma_{3} I_{z}}{m \Gamma_{2}} .
$$

The conditions (42), (45) are defined for the time-invariant case, which may seem reasonable at each time step with constant speed, but the system is time-varying in practice. Consequently, the following two subsections investigate the stability of the parameter-varying lateral dynamics with the pure/combined slip LuGre tire model using the quadratic stability approach for LPV systems. 


\section{B. Quadratic Stability}

The quadratic stability of the system is analyzed in this section. The linear parts of (16) and (35) are in the following form

$$
\dot{x}(t)=A(\omega) x(t),
$$

which is an LPV system due to the time-varying angular velocity $\omega(t)$. Thus, evaluating the eigenvalues does not lead to asymptotic stability. Instead, the following proposition is valid

Proposition 1: [35] The following linear time-varying system

$$
\dot{x}(t)=G(t) x(t),
$$

is exponentially stable if $\bar{\lambda}<0$, with a convergence rate of at least $|\bar{\lambda}|$, where

$$
\bar{\lambda}=\max _{i, t \geq 0} \lambda_{i}\left(\frac{G(t)+G^{T}(t)}{2}\right)
$$

According to Proposition 1, system (46) is exponentially stable if $\lambda_{i}(A(\omega(t)))<0$ for all $t \geq 0$. Thus, by a converse theorem for Lyapunov stability [36], one can conclude that there exists a quadratic Lyapunov function for (46). However, for the simplicity of the linear system, the quadratic Lyapunov candidate is assumed to be in the form of $V(x(t))=\frac{1}{2} x(t)^{T} P x(t)$ for some $P>0$. The derivative of the Lyapunov function along the state trajectories leads to the following condition for some $Q>0$ :

$$
\begin{aligned}
\dot{V}(x(t)) & =(A x(t))^{T} P x(t)+x(t)^{T} P(A x(t)) \\
& =-x(t)^{T} Q x(t),
\end{aligned}
$$

This can be written as

$$
\begin{aligned}
\dot{V}(x(t)) & =-x(t)^{T} Q x(t) \leq-\lambda_{\min }(Q) x(t)^{T} x(t) \\
& \leq-\frac{\lambda_{\min }(Q)}{\lambda_{\max }(P)} x(t)^{T} P x(t)=-\alpha V(x(t)),
\end{aligned}
$$

where $\alpha=\frac{\lambda_{\min }(Q)}{\lambda_{\max }(P)}>0$. By choosing $P=I$, one gets $Q(\omega)=-\frac{1}{2}\left(A(\omega)+A(\omega)^{T}\right)$, which is the same as the matrix introduced in Proposition 1. Matrix $Q(\omega)$ is the symmetric part of $A(\omega)$, and from now, it is denoted by $A_{s}(\omega)$. Note that the stability of $A_{s}(\omega(t))$ is a sufficient condition only for the stability of (46). In other words, the system (46) may be stable while $A_{s}(\omega)$ is unstable. To find a physical condition (interpretation) for the current time-varying linear lateral models (17) and (36) similar to the condition (4) in the 2DOF bicycle model, the stability of the symmetric $A_{s}(\omega)=$ $\frac{A(\omega)+A^{T}(\omega)}{2}$ and $A_{s}^{\prime}(\omega)=\frac{A^{\prime}(\omega)+A^{\prime T}(\omega)}{2}$ is studied in this subsection for both the pure and combined-slip approaches.

1) Pure-slip model:: For the stability of $A_{s}(\omega)_{2 \times 2}$, it is necessary and sufficient to have $\operatorname{Tr}\left(A_{s}\right)<0$ and $\operatorname{Det}\left(A_{s}\right)>$ 0 for all $t \geq 0$. Satisfying these two conditions leads to the following longitudinal speed criteria:

$$
\left|\frac{\chi_{4}}{u}-\frac{u}{2}\right|<\frac{\sqrt{\chi_{1} \chi_{3}}}{u}
$$

where $\chi_{4}=\frac{a b g}{2 l}\left(\frac{\sigma_{0 r}}{\kappa_{r}}-\frac{\sigma_{0 f}}{\kappa_{f}}\right)\left(1+\frac{m}{I_{z}}\right)$.
2) Combined friction model: The same methodology used for stability of the assumed time-varying symmetric matrix $A_{s}^{\prime}$ is used for the combined-slip case that leads to:

$$
\left|\frac{\Gamma_{4}}{u}-\frac{u}{2}\right|<\frac{\sqrt{\Gamma_{1} \Gamma_{3}}}{u},
$$

in which $\Gamma_{1}, \Gamma_{3}, \Gamma_{4}$ are provided in (44) and

$$
\Gamma_{4}=\frac{a b g}{2 l}\left(1+\frac{m}{I_{z}}\right)\left(s_{r l}+s_{r r}-s_{f l}-s_{f r}\right)
$$

One can compare the criteria (50) for LPV pure and combined-slip systems with their counterparts (42) for timeinvariant systems and with (4) for the bicycle model. However, the stability of $A_{s}(\omega)$ and $A_{s}^{\prime}(\omega)$ is a conservative sufficient condition for the stability of (46). Consequently (50) and (51) for both pure and combined-slip cases, suggest conservative conditions for the maximum longitudinal speed. The following section provides another approach for the stability of (46), which consequently reaches a less conservative limit on $u$. Limits for the speed and slip ratio based on the quadratic stability of the time-varying pure/combined-slip models are listed in Tables III, IV. These limits are compared with the affine quadratic stability case be studied in the next section.

\section{Affine Quadratic Stability}

To find a less conservative condition than the asymptotic stability of the symmetric matrix $A_{s}(\omega)$ and to check the possibility of stability at greater speeds, the affine quadratic stability of linear parts of the time-varying systems (16), (35) is studied here, using the notation of (46) with the initial condition $x(0)=x_{0}$ for all $t \geq 0$. The state matrix $A(\omega)$ is said to be affinely dependent on the parameters $\omega$ when known and fixed matrices $A_{0}$ and $A_{1}$ exist such that $A(\omega)=$ $A_{0}+\omega A_{1}$. The time-varying parameter and its derivatives are in the sets $\omega_{p} \in\left[\omega_{l}, \omega_{u}\right]$ and $\dot{\omega}_{p} \in\left[\dot{\omega}_{l}, \dot{\omega}_{u}\right]$ respectively.

Theorem 1: [37] The linear system (46) is affinely quadratically stable over all possible trajectories of the parameter vector $\omega(t)$ if $A\left(\omega_{m}\right)$ is stable ( $\omega_{m}$ is the average of parameter span) and there exists an affine positive definite Lyapunov function $V(x, \omega)=x^{T} P(\omega) x$ with $P(\omega)=$ $P_{0}+\omega P_{1}>0$ such that $d V(\omega, \dot{\omega}) / d t<0$ for all initial conditions $x_{0}$ and the additional multi-convexity constraint $A_{1}^{T} P_{1}+P_{1} A_{1} \geq 0$ holds [38]. The condition $\dot{V}<0$ resembles

$$
A^{T}\left(\omega_{p}\right) P\left(\omega_{p}\right)+P\left(\omega_{p}\right) A\left(\omega_{p}\right)+P\left(\dot{\omega}_{p}\right)-P_{0}<0
$$

for all $(\omega, \dot{\omega}) \in \mathcal{S}_{p} \times \mathcal{S}_{r}$ where $\mathcal{S}_{p}$ is the set of vertices of the parameter box $\left[\omega_{l}, \omega_{u}\right]$ and $\mathcal{S}_{r}$ is the set of corners of the rates $\left[\dot{\omega}_{l}, \dot{\omega}_{u}\right]$. The affine quadratic stability condition implements the variation rate $\dot{\omega}(t)$, which makes it less conservative than the quadratic stability criteria.

Proposition 2: The linear part of the lateral dynamics (16) and (35) are affinely quadratically stable with respect to the parameter $\omega(t) \in\left[\omega_{l}, \omega_{u}\right]$ for all $t \geq 0$ for some $\omega_{l}, \omega_{u}$. 
1) Pure-slip model: To show the above proposition for the pure-slip model, we rewrite the state matrix $A(\omega)$ in an affinely dependent form, and we use $k_{i} \approx \frac{\sigma_{0 i}}{\kappa_{i} R_{e} \omega_{i}}$ since $\sigma_{2}$ is small for both front and rear tires:

$$
A(\bar{\omega})=\left[\begin{array}{cc}
0 & -u \\
0 & 0
\end{array}\right]+\bar{\omega}\left[\begin{array}{cc}
-\frac{\chi_{1}}{R_{e}} & \frac{2 \chi_{4} I_{z}}{\left(m+I_{z}\right) R_{e}} \\
\frac{2 m \chi_{4}}{\left(m+I_{z}\right) R_{e}} & -\frac{\chi_{3}}{R_{e}}
\end{array}\right]
$$

where $\bar{\omega}=\frac{1}{\omega_{i}}$. The introduced wheel speed $\bar{\omega}$ is a bounded variable with an operating region of $\bar{\omega} \in\left[\frac{1}{\omega_{u}}, \frac{1}{\omega_{l}}\right]$. The rate of variation is also bounded between $\dot{\bar{\omega}} \in\left[\frac{1}{\dot{\omega}_{u}}, \frac{1}{\dot{\omega}_{u}}\right]$. The affine matrix $P(\omega)=P_{0}+\omega_{1} P_{1}$ can be found numerically, such that (53) holds and $A_{1}^{T} P_{1}+P_{1} A_{1} \geq 0$. LMIs (53) are solved with Matlab, and the stability of the linear part of the system (16) is guaranteed with the substitution of the operating region $\|\omega\| \leq 160[\mathrm{rad} / \mathrm{s}]$ and the rate of change $\|\dot{\bar{\omega}}\| \leq 700\left[\mathrm{rad} / \mathrm{s}^{2}\right]$ with sampling frequency $200[\mathrm{~Hz}]$.

2) Combined-slip condition: Affine quadratic stability can also be investigated for the combined-slip model, as shown in the following paragraph. Equation (36) can be rewritten as an affine linear parameter varying system such as $A(\bar{\omega})=A_{0}+\bar{\omega}_{f l} A_{1}+\bar{\omega}_{f r} A_{2}+\bar{\omega}_{r l} A_{3}+\bar{\omega}_{r r} A_{4}$ where $\bar{\omega}_{i}=1 / \omega_{i}$. With $\mathcal{G}_{i}=\frac{\sigma_{0 i}^{\prime} \rho_{i}^{\prime}}{\eta_{i} R_{e} \sigma_{0 i}\left|\lambda_{i}\right|+\kappa R_{e} \rho_{i}^{\prime}}, A(\bar{\omega})$ can be expressed as:

$$
\begin{aligned}
A(\bar{\omega}) & =\left[\begin{array}{cc}
0 & -u \\
0 & 0
\end{array}\right]+\sum_{i_{1}} \bar{\omega}_{i_{1}} \frac{b g}{2 l} \mathcal{G}_{i_{1}}\left[\begin{array}{cc}
-1 & -a \\
-\frac{m a}{I_{z}} & -\frac{m a^{2}}{I_{z}}
\end{array}\right] \\
& +\sum_{i_{2}} \bar{\omega}_{i_{2}} \frac{a g}{2 l} \mathcal{G}_{i_{2}}\left[\begin{array}{cc}
-1 & b \\
\frac{m b}{I_{z}} & -\frac{m b^{2}}{I_{z}}
\end{array}\right]
\end{aligned}
$$

in which $i_{1} \in\{f l, f r\}, i_{2} \in\{r l, r r\}$.

\section{RESUlts AND Discussion}

Independent simulations are carried out in this section using the steady-state and transient LuGre tire models with the pure and combined-slip assumption. Stability of the suggested parameter-varying lateral models is also explored. The vehicle selected for the first test is a Sedan with the specifications listed in Table I. A standard double lane change maneuver with the maximum steer angle of $\delta=0.0525[\mathrm{rad}]$ on the front wheels is examined and the lateral velocity response with $u=70[\mathrm{kph}]$ on a dry road is depicted in Fig. 7. The response of the system with the linear tire model is compared to the complete (both linear and nonlinear parts) steady state and transient pure-slip models (16), (29).

As can be seen in Fig. 7, the steady-state model demonstrates good accuracy (compared with the transient one). The predicted larger lateral velocity for the LuGre models is due to the fact that the saturation, nonlinear regions, and consequently, tire capacities are considered in these approaches, but the linear tire model has no capacity or limit on lateral forces.

The pure-slip model (5) can also be employed for the longitudinal direction. The performance of the pure-slip longitudinal LuGre model is studied in Fig. 8. The maneuver is successive acceleration and brake with initial speed of $u=40[k p h]$ and no steering on dry road. The longitudinal

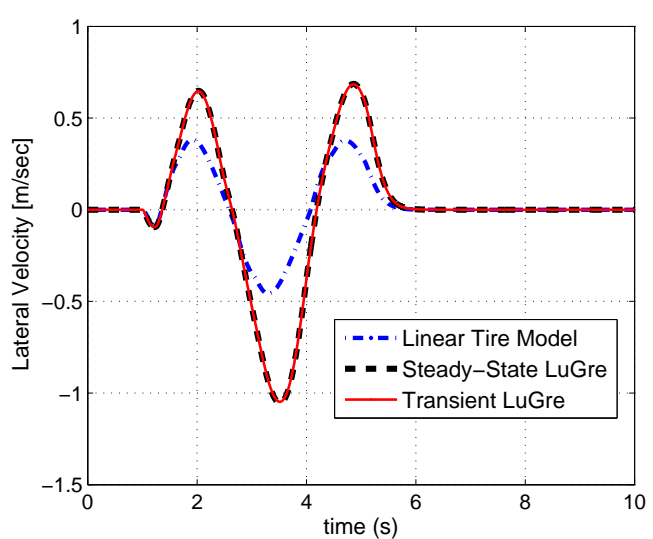

Fig. 7: Lateral velocity, pure-slip SS and transient model

forces with the pure-slip LuGre model on the front left tire are depicted in Fig. 8.

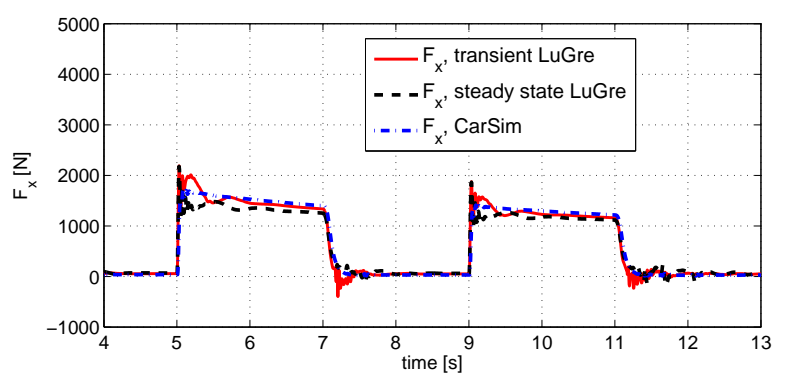

Fig. 8: Transient vs. steady state model, longitudinal forces in acceleration/brake

There are deviations between the steady-state and transient model near the acceleration and brake points due to not considering the transient response in the steady-state approach, but the performance of the steady-state model is good as quantified in Table II for different maneuvers and vehicles. To summarize, the RMS of the error between the transient and steady-state LuGre models are listed in Table II for two vehicle models. The step steer and double lane change maneuvers are denoted by S-Str and DLC, respectively. Deviations of the steady-state (SS) front tire internal state $z_{y f}$ and the slip angle $\alpha_{f}$ from the transient ones with the pure-slip condition are reported in Table II as well.

TABLE II: Steady State Model Error RMS

\begin{tabular}{ccc}
\hline Maneuver & $v$ & $r$ \\
\hline SUV; S-Str $\delta=0.03, u_{x}=60 k p h$ & $0.21 \%$ & $0.23 \%$ \\
SUV; S-Str $\delta=0.03, u_{x}=90 k p h$ & $0.57 \%$ & $1.2 \%$ \\
Sedan; DLC $\delta=0.052, u_{x}=80 k p h$ & $0.8 \%$ & $1.64 \%$ \\
Sedan; DLC $\delta=0.075, u_{x}=80 k p h$ & $2.17 \%$ & $2.6 \%$ \\
\hline Maneuver & $z_{y f}$ & $\alpha_{f}$ \\
\hline SUV; S-Str $\delta=0.03, u_{x}=70 k p h$ & $0.4 \%$ & $0.36 \%$ \\
Sedan; DLC $\delta=0.03, u_{x}=80 k p h$ & $0.6 \%$ & $0.72 \%$ \\
Sedan; DLC $\delta=0.075, u_{x}=80 k p h$ & $1.47 \%$ & $1.6 \%$ \\
\hline
\end{tabular}

As seen in Table II, the SS model exhibits good accuracy in slip angle and $z_{y f}$, which are correlated with the lateral 
states $v$ and $r$. It substantiates that the states calculation error produced by the SS model with the pure-slip assumption is negligible in such DLC and S-Str driving scenarios. To compare the results of the linear part of the suggested pureslip model (16) (PS, Lin. LuGre), the linear part of the proposed combined-slip model (35) (CS, Lin. LuGre), the complete form (linear and nonlinear) of (16) (PS, NonLin. LuGre), and the conventional linear bicycle model (3) (Linear tire), a standard double-lane change maneuver is performed on the SUV with parameters provided in Table I. The lateral velocity results of the test on a dry road with speed $u=$ $54[k p h]$ are illustrated in Fig. 9. The maximum steering angle is $\delta_{\max }=0.07[\mathrm{rad}]$ on the front wheels, and the longitudinal slip ratio is $\lambda=20 \%$ for the combined-slip model.

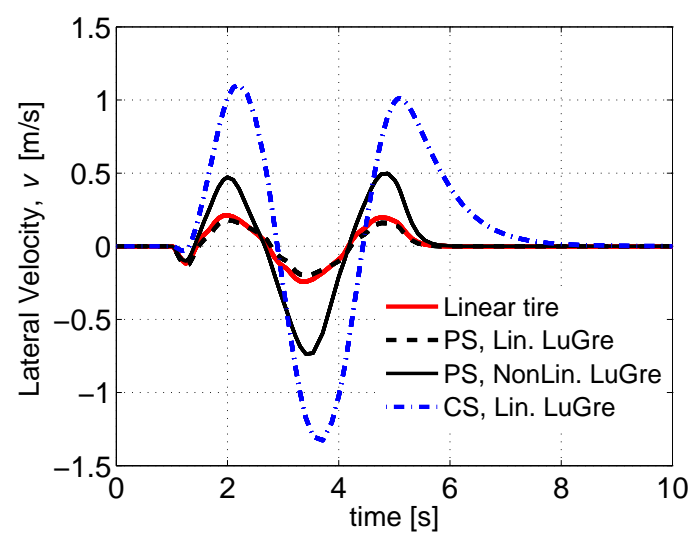

Fig. 9: Double-lane change, LuGre vs. the linear tire model

It can be concluded from Fig. 9 that the linear tire model provides the same response as the linear part of (16). Thus, the tuned LuGre parameters in Table I are compatible with the cornering stiffness of the linear tire model. This compatibility is also confirmed by Fig. .11, which shows correspondence between the experimental tire curve and the LuGre model with the identified parameters in Table I. As predicted by the model, the slip ratio significantly affects tire capacity and results in higher lateral velocity. The expected lateral tire forces of the combined-slip model, even with the linear part, are smaller than those of the complete LuGre (linear and nonlinear) model with pure-slip assumption. Thus, the predicted largest vehicle's side-slip angle $\beta$ at CG for the combined-slip model with the linear part is $\beta_{c s, l i n}=5.15^{\circ}$, which is higher than the acceptable margin $\beta=5^{\circ}$ and suggests marginally stable condition. On the other hand, the complete form (linear and nonlinear) of the pure-slip LuGre model exhibits $\beta_{p s, \text { nonlin }}=2.7^{\circ}$ that is within the allowable side-slip angle margin.

Figure 10 illustrates the lateral velocity of the same SUV using the developed pure and combined-slip models (denoted by PS and CS in the figure) on a slippery road with $\theta=0.4$ and speed $u=47[\mathrm{kph}]$. The combined-slip results are plotted as well for the longitudinal slip ratio $\lambda=25 \%$.

Figure 10 confirms the reducing effect of the longitudinal slip ratio on the lateral capacity of tires. A higher slip ratio

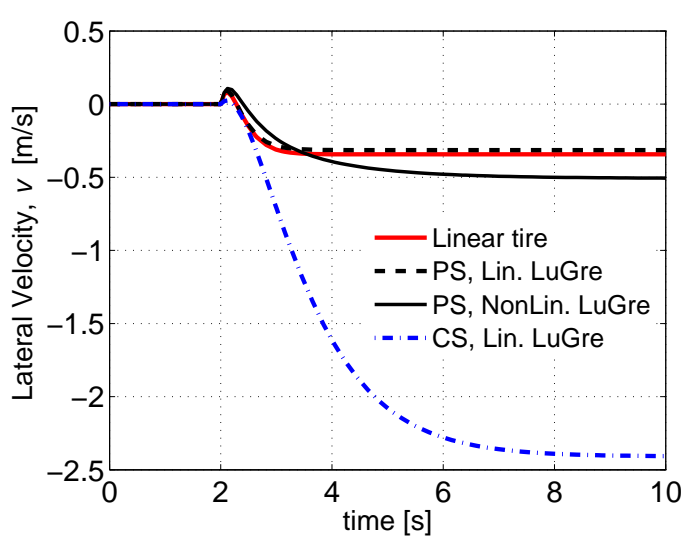

Fig. 10: Step steer, pure/combined-slip LuGre vs. the linear tire on a slippery road $\theta=0.4$

reduces the tires' lateral forces and leads to higher lateral velocity and consequently large and unstable maximum sideslip angle $\beta_{C S, \text { Lin }}=10.6^{\circ}$ on the slippery road.

Example 1: The SUV with the parameters introduced in Table I is selected here to show the difference between the outcome of the quadratic stability and the stability of the symmetric matrix $A_{s}(t)$. The critical speed obtained by (50), which is the location of the eigenvalues of symmetric matrix $A_{s}(t)$ is $u=12.4 \mathrm{~m} / \mathrm{s}$ on a dry road, but the affine quadratic stability condition (53) with the multi-convexity constraint $a_{i}^{T} P_{i}+P_{i} a_{i} \geq 0$ and $P(\omega)>0$ suggests $u=39.1 \mathrm{~m} / \mathrm{s}$ with:

$$
P(\bar{\omega})=\left[\begin{array}{cc}
0.39-0.37 \bar{\omega} & 0.008-0.009 \bar{\omega} \\
0.008-0.009 \bar{\omega} & 0.024-0.014 \bar{\omega}
\end{array}\right]
$$

This confirms that the affine quadratic stability provides a more practical stability condition than the stability of the symmetric matrix $A_{s}(t)$, which is conservative. The maximum allowable speed are compared for the three stability criteria in Table III in which $L T I, L G$ and $L T I$, L-tire represent the time-invariant stability conditions (42) and (4) on the lateral dynamics with the linear LuGre and conventional linear tire models respectively. The quadratic and affine quadratic stability conditions are denoted by $Q S$ and $A Q S$ respectively.

TABLE III: Speed limits for the pure-slip case

\begin{tabular}{ccccc}
\hline Maneuver & LTI, L-tire & LTI, $L G$ & $Q S$ & $A Q S$ \\
\hline Dry road $\theta=1$ & $66 \mathrm{~m} / \mathrm{s}$ & $63.8 \mathrm{~m} / \mathrm{s}$ & $11.3 \mathrm{~m} / \mathrm{s}$ & $42 \mathrm{~m} / \mathrm{s}$ \\
Dry road $\theta=0.5$ & $46.7 \mathrm{~m} / \mathrm{s}$ & $44.7 \mathrm{~m} / \mathrm{s}$ & $7.9 \mathrm{~m} / \mathrm{s}$ & $29.4 \mathrm{~m} / \mathrm{s}$ \\
\hline
\end{tabular}

Example 2: The stability of the symmetric matrix $A_{s}^{\prime}(t)$ and the quadratic stability criteria of the affine system (55) are compared in this example. The same vehicle is tested with the combined-slip LuGre mode. The affine quadratic stability condition (53) on the affine system (55) (with independent wheel speed at each corner) suggests the following symmetric matrix $P\left(\bar{\omega}_{i}\right)$ with $p_{1}=1.84-1.03 \bar{\omega}_{f}+0.0007 \bar{\omega}_{r}$, $p_{2}=-0.29-0.065 \bar{\omega}_{f}+0.53 \bar{\omega}_{r}$ and $p_{3}=0.845-0.56 \bar{\omega}_{f}+$ 
$0.254 \bar{\omega}_{r}$ on a dry road with $\lambda=20 \%$ :

$$
P(\bar{\omega})=\left[\begin{array}{ll}
p_{1}\left(\bar{\omega}_{i}\right) & p_{2}\left(\bar{\omega}_{i}\right) \\
p_{2}\left(\bar{\omega}_{i}\right) & p_{3}\left(\bar{\omega}_{i}\right)
\end{array}\right]
$$

in which $\bar{\omega}_{f}=\bar{\omega}_{f l}+\bar{\omega}_{f r}, \bar{\omega}_{r}=\bar{\omega}_{r l}+\bar{\omega}_{r r}$. This stability criteria suggests the maximum speed $u=19.6[\mathrm{~m} / \mathrm{s}]$, but the stability of the symmetric matrix $A_{s}^{\prime}(t)$ yields the maximum speed $u=8.1[\mathrm{~m} / \mathrm{s}]$. The maximum allowable longitudinal slip ratio $\lambda_{\max }$ and the maximum speed are compared for the two stability criteria in Table IV.

TABLE IV: Speed and $\lambda$ limits for the combined-slip

\begin{tabular}{ccc}
\hline Maneuver & $Q S$ & $A Q S$ \\
\hline Dry road $\theta=1, u=10 \mathrm{~m} / \mathrm{s}$ & $\lambda_{\max }=15 \%$ & $\lambda_{\max }=34 \%$ \\
Wet road $\theta=0.5, u=8 \mathrm{~m} / \mathrm{s}$ & $\lambda_{\max }=31 \%$ & $\lambda_{\max }=49 \%$ \\
Dry road $\theta=1, \lambda=20 \%$ & $u_{\max }=8.1 \mathrm{~m} / \mathrm{s}$ & $u_{\max }=19.6 \mathrm{~m} / \mathrm{s}$ \\
Wet road $\theta=0.5, \lambda=40 \%$ & $u_{\max }=5.7 \mathrm{~m} / \mathrm{s}$ & $u_{\max }=13.8 \mathrm{~m} / \mathrm{s}$ \\
\hline
\end{tabular}

The first two rows provide the limit for the longitudinal slip ratio, $\lambda_{\max }$, on both dry and wet roads, with constant speed. The maximum speed, $u_{\max }$, for a constant slip ratio on different roads is suggested in the third and fourth rows. Table IV reveals the conservative characteristics of the stability criteria on $A_{s}^{\prime}(t)$

\section{CONCLUSION}

The purpose of this paper has to address the nonlinear and saturated tire behaviors as well as linear region in lateral vehicle dynamics. Two main developments have been adopted for the tire-vehicle lateral state space model: a) incorporation of both steady-state and transient LuGre tires; b) employing the pure and combined-slip models. Based on the developed tire-vehicle lateral dynamics and simulation results, the following conclusions can be made:

Application to control/estimation: the novel methodology presented in this article and the suggested general forms of the tire-vehicle lateral models (16) and (35) provide a framework to achieve analytical solutions for control and estimation problems. This has a significant advantage over the cascaded methods that need slip ratio/angles to calculate forces (in a tire model) and then to get vehicle states by solving lateral dynamics and calculated forces.

Transient vs. steady-state LuGre model: A closed form solution for the transient LuGre model was developed in (29). This solution converges to the steady state LuGre model response with negligible deviations as shown in Tables II. The steady state LuGre model can be used effectively in vehicle stability analysis with insignificant impact on the results.

Lateral dynamics with the pure/combined-slip models: The interaction of longitudinal and lateral slips and their effect on the lateral dynamics were fully studied in this article. Equation (16) provides a general form of the pure-slip lateral dynamics in which the linear part is isolated from the nonlinear. The vehicle dynamics assuming a combined tire slip is presented in (35). This equation can be used to predict accurately the vehicle's lateral stability when tires are in their nonlinear and saturated regions. Therefore, controllers and state observers can be designed based on the closed formulations (16) and (35) of the tire-vehicle model delivered in this paper.

Normal force incorporation at each corner: one significant advantage of the suggested model is that it can be used whenever normal forces at each corner are available; see (20) for the pure-slip case and (39) for the combined-slip friction model.

Large slip angles: for large slip angle cases, the nonlinear part $H(t)$ in (16) yields a form that contains states (lateral velocity and yaw rate). Thus, the general form (linear and nonlinear parts) of the pure-slip condition can be rewritten in a linear parameter-varying system provided in (26), which is practical for the stability analysis or control applications.

Stability, time-invariant case: different stability criteria for the derived lateral dynamics were explored and compared with that of the bicycle model. For the time-invariant case, speed limit criteria (42) and (45) are suggested for the pure and combined-slip cases respectively.

Stability, time-varying case: The quadratic stability (QS) of the linear parts of the time-varying pure/combined-slip lateral dynamics was investigated and speed limits for the pure and combined-slip models were suggested in (50) and (51). Furthermore, the time-varying systems (16), (35) were rewritten in the affine parameter-varying forms (54), (55) with a proper variable change and affine quadratic stability (AQS) was studied to find a less conservative condition than the QS. The AQS suggests more practical speed limits guaranteeing the quadratic stability of the proposed tirevehicle lateral model.

\section{APPENDIX}

\section{A. LuGre parameter identification}

The LuGre tire parameters are obtained by fitting to the experimental tire curves with the Nonlinear Least Square method and the result is shown in Fig. .11 for the normal force of $F_{z}=4.5[k N]$ on a dry road. Identified parameters are also listed in Table I

\section{B. Derivation of Eq. (11)}

The lateral internal state of the LuGre tire model for the steady-state $\dot{\bar{z}}_{y}=0$ pure-slip condition can be written as:

$$
\bar{z}_{y}=\frac{v_{r y}}{\frac{\sigma_{0 y}\left|v_{r y}\right|}{\theta g\left(v_{r y}\right)}+\kappa R_{e} \omega}
$$




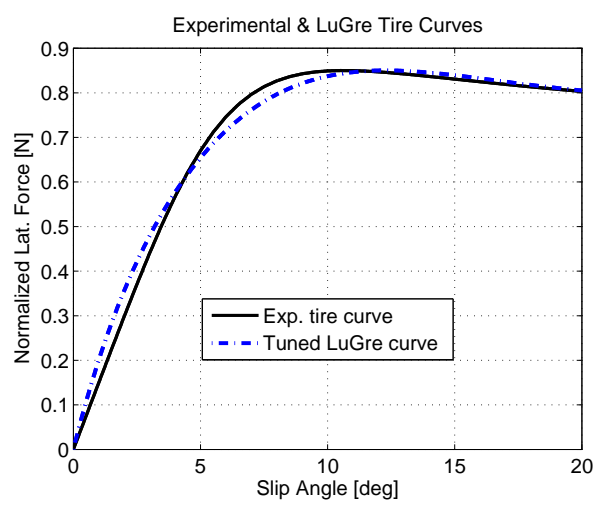

Fig. .11: Lateral LuGre tire curve and the experimental tire curve

The relative lateral velocity is $v_{r y}=u \alpha$. The the normalized lateral force yields:

$$
\begin{aligned}
\mu_{y} & =\sigma_{0 y} \bar{z}_{y}+\sigma_{2 y} v_{r y} \\
& =\left(\frac{1}{\frac{u|\alpha|}{\theta g\left(v_{r y}\right)}+\frac{\kappa R_{e} \omega}{\sigma_{0 y}}}+\sigma_{2 y}\right) u \alpha \\
& =\left(\frac{\rho / u}{|\alpha|+\gamma \rho / u}+\sigma_{2 y}\right) u \alpha
\end{aligned}
$$

where $\sigma_{0 y}$ and $\sigma_{2 y}$ are rubber stiffness and relative viscous damping defined in Table I, $\rho=\theta g\left(v_{r y}\right)$, and $\gamma=$ $\kappa R_{e} \omega / \sigma_{0 y}$.

\section{Lateral dynamics with the transient LuGre model}

The lumped LuGre model (5) can be written as:

$$
\begin{aligned}
\dot{\bar{z}}_{y} & =v_{r y}-\left(\frac{\sigma_{0 y}\left|v_{r y}\right|}{\theta g\left(v_{r y}\right)}+\kappa R_{e}|\omega|\right) \bar{z}_{y} \\
& =-\left(\sigma_{0 y} \gamma+\frac{\sigma_{0 y} u|\alpha|}{\rho}\right) \bar{z}_{y}+v_{r y} \\
& =-\frac{\sigma_{0 y} u}{\rho}\left(\frac{\gamma \rho}{u}+|\alpha|\right) \bar{z}_{y}+v_{r y} \\
& =-\mathcal{E}(t) \bar{z}_{y}+v_{r y}
\end{aligned}
$$

which has the solution $\bar{z}_{y}=\int_{0}^{t} e^{-\mathcal{E}(\tau)(t-\tau)} v_{r y} d \tau$, assuming zero initial condition. After integrating and substituting $\bar{z}_{y}$ and $\dot{\bar{z}}_{y}$ into the normalized force equation $\mu=\sigma_{0 y} \bar{z}_{y}+$ $\sigma_{1 y} \dot{\bar{z}}_{y}+\sigma_{2} v_{r y}$, the following lateral normalized force will be obtained for the transient LuGre model:

$$
\begin{aligned}
\mu_{G y i} & =e^{-\sigma_{0}\left(\gamma_{i}+\frac{u\left|\alpha_{i}\right|}{\rho_{i}}\right)}\left(\sigma_{1 i}-\frac{1}{\gamma_{i}+\frac{u\left|\alpha_{i}\right|}{\rho_{i}}}\right) u \alpha_{i} \\
& +\frac{u \alpha_{i}}{\gamma_{i}+\frac{u\left|\alpha_{i}\right|}{\rho_{i}}}+\sigma_{2 i} u \alpha_{i} .
\end{aligned}
$$

The linear part is proposed is extracted as follows with the road condition $\theta$ and the slip angle $\alpha_{i}$ at each tire.

$$
\mu_{y i}=\left[\frac{1}{\gamma_{i}}+e^{-\kappa_{i} R_{e} \omega t}\left(\sigma_{1 i}-\frac{1}{\gamma_{i}}\right)+\sigma_{2 i}\right] \theta u \alpha_{i} .
$$

Thus, the nonlinear part can be described as

$$
\begin{aligned}
\bar{\phi}_{i} & =e^{-\sigma_{0 i} \gamma_{i} t}\left[\left(\sigma_{1 i}-\frac{\rho_{i}}{u\left|\alpha_{i}\right|+\gamma_{i} \rho_{i}}\right) e^{\frac{-\sigma_{0 i} u\left|\alpha_{i}\right| t}{\rho_{i}}}\right] u \alpha_{i} \\
& +\left(\frac{\rho}{u|\alpha|+\rho \gamma}-\frac{\theta}{\gamma}\right) u \alpha_{i} \\
& -e^{-\sigma_{0 i} \gamma_{i} t} \theta\left(\sigma_{1 i}-\frac{1}{\gamma_{i}}\right) u \alpha_{i} .
\end{aligned}
$$

Therefore, $\bar{\phi}_{i}$ can be defined as $\bar{\phi}_{i}=\phi_{i}+\Phi_{i}$ where $\phi_{i}$ and $\Phi_{i}$ are provided in (19) and (30) respectively. Substituting the general form of the transient LuGre normalized force (C.2) into the lateral dynamics (2) yields the following lateral state representation:

$$
\begin{aligned}
{\left[\begin{array}{c}
\dot{v} \\
\dot{r}
\end{array}\right] } & =\left[\begin{array}{cc}
\frac{-g}{l}\left(b \bar{k}_{f}+a \bar{k}_{r}\right) & -\left(\frac{a b g}{l}\left(\bar{k}_{f}-\bar{k}_{r}\right)+u\right) \\
\frac{m a b g}{I_{z} l}\left(\bar{k}_{r}-\bar{k}_{f}\right) & -\frac{m a b g}{I_{z} l}\left(b \bar{k}_{r}+a \bar{k}_{f}\right)
\end{array}\right]\left[\begin{array}{c}
v \\
r
\end{array}\right] \\
& +\left[\begin{array}{c}
\frac{b g}{l} \bar{k}_{f} u \\
\frac{m a b g}{I_{z} l} \bar{k}_{f} u
\end{array}\right] \delta+\left[\begin{array}{c}
\frac{g u}{l}\left(b \bar{\phi}_{f} \alpha_{f}+a \bar{\phi}_{r} \alpha_{r}\right) \\
\frac{m a b g u}{I_{z} l}\left(\bar{\phi}_{f} \alpha_{f}-\bar{\phi}_{r} \alpha_{r}\right)
\end{array}\right] .
\end{aligned}
$$

where $\bar{k}_{f}$ and $\bar{k}_{r}$ are defined in (29) for the front and rear axles.

\section{REFERENCES}

[1] H. Pacejka and R. Sharp, "Shear Force Development by Pneumatic Tyres in Steady State Conditions: A Review of Modelling Aspects,' Vehicle System Dynamics, vol. 20, no. 3-4, pp. 121-175, 1991.

[2] H. B. Pacejka and I. J. M. Besselink, "Magic Formula Tyre Model with Transient Properties," Vehicle System Dynamics, vol. 27, pp. 234-249, 1997.

[3] R. Uil, "Tyre Models for Steady-State Vehicle Handling Analysis," Ph.D. dissertation, Eindhoven University of Technology, 2007.

[4] C. C. De Wit, H. Olsson, K. J. Astrom, and P. Lischinsky, "A new model for control of systems with friction," IEEE Transactions on automatic control, vol. 40, no. 3, pp. 419-425, 1995.

[5] C. Canudas-de wit and R. Horowitz, "Observer for Tire/Road Contact Friction Using Only Wheel Angular Velocity Information," in 38th Conference on Decision \& Control, 1999, pp. 3932-3937.

[6] C. Canudas-De-Wit and P. Tsiotras, "Dynamic Tire Friction Models for Vehicle Traction Control," in proceedings of the 38th Conference on Decision \& Control, 1999, pp. 3746-3751.

[7] C. Canudas-de Wit, M. L. Petersen, and A. Shiriaev, "A new nonlinear observer for tire/road distributed contact friction," in Decision and Control, 2003. Proceedings. 42nd IEEE Conference on, vol. 3. IEEE, 2003, pp. 2246-2251.

[8] P. Tsiotras, E. Velenis, and M. Sorine, "A LuGre Tire Friction Model With Exact Aggregate Dynamics," Vehicle System Dynamics, vol. 42, pp. 195-210, 2004.

[9] E. Velenis, "Extension of the lugre dynamic tire friction model to 2D motion," Proceedings of the 10th IEEE Mediterranean Conference on Control and Automation, 2002.

[10] J. Deur, J. Asgari, and D. Hrovat, "A 3D Brush-Type Dynamic Tire Friction Model," Vehicle System Dynamics, vol. 42, no. 3, pp. 133 173, 2004.

[11] W. Liang, J. Medanic, and R. Ruhl, "Analytical Dynamic Tire Model,' Vehicle System Dynamics, vol. 46, pp. 197-227, 2008.

[12] J. Y. Wong, Theory of Ground Vehicles, fourth ed. ed. John Wiley Sons, 2008.

[13] R. Rajamani, Vehicle dynamics and control. Springer Science Business Media, 2011

[14] V. Nguyen, "Vehicle handling, stability, and bifurcation analysis for nonlinear vehicle models," Master's thesis, University of Maryland, College Park, 2005.

[15] L. Li, F. Y. Wang, and Q. Zhou, "Integrated Longitudinal and Lateral Tire/Road Friction Modeling and Monitoring for Vehicle Motion Control," IEEE Transactions on Intelligent Transportation Systems, no. 1, pp. 1-19, 2006. 
[16] D. C. Liaw, H. H. Chiang, and T. T. Lee, "A Bifurcation Study of Vehicle's Steering Dynamics," in IEEE Proceedings of Intelligent Vehicles Symposium, 2005, pp. 388-393.

[17] H. H. C. D. C. Liaw and T. T. Lee, "Elucidating vehicle lateral dynamics using a bifurcation analysis," IEEE Transaction on Intelligent Transportation Systems, vol. 8, no. 1, 2007.

[18] S. Shen, J. Wang, P. Shi, and G. Premier, "Nonlinear dynamics and stability analysis of vehicle plane motions," Vehicle System Dynamics, vol. 45, no. 1, pp. 15-35, 2007.

[19] L. J. S. S. X. Wang and L. Liu, "Analysis of driving mode effect on vehicle stability," International Journal of Automotive Technology, vol. 14, no. 3, pp. 363-373, 2013.

[20] G. M. F. Della Rossa and C. Piccardi, "Bifurcation analysis of an automobile model negotiating a curve," Vehicle System Dynamics, vol. 50, pp. 1539-1562, 2012.

[21] T. D. Gillespie, Fundamental of Vehicle Dynamics. Society of Automotive Engineers, 1992.

[22] S. Antonov, A. Fehn, and A. Kugi, "Unscented kalman filter for vehicle state estimation," Vehicle System Dynamics, vol. 49, no. 9, pp. 14971520, 2011.

[23] X. Zhang, Y. Xu, M. Pan, and F. Ren, "A vehicle ABS adaptive slidingmode control algorithm based on the vehicle velocity estimation and tyre/road friction coefficient estimations," Vehicle System Dynamics, vol. 52, no. 4, pp. 475-503, 2014.

[24] M. Gadola, D. Chindamo, M. Romano, and F. Padula, "Development and validation of a kalman filter-based model for vehicle slip angle estimation," Vehicle System Dynamics, vol. 52, no. 1, pp. 68-84, 2014.

[25] E. Hashemi, A. Kasaeizadeh, A. Khajepour, N. Mushchuk, and S.-K. Chen, "Robust Estimation and Experimental Evaluation of Longitudinal Friction Forces in Ground Vehicles," in ASME IMECE2014, 2014.

[26] K. J. Astrom and C. Canudas-de Wit, "Revisiting the LuGre Model," IEEE Control Systems Magazine, vol. 6, pp. 101-114, 2008.

[27] C. Canudas-de Wit, P. Tsiotras, E. Velenis, M. B. Gissinger, and G. Gissinger, "Dynamic Friction Models for Road/Tire Longitudinal Interaction," Vehicle System Dynamics, vol. 39, pp. 189-226, 2003.

[28] E. Hashemi, M. Pirani, A. Khajepour, B. Fidan, A. Kasaiezadeh, S. Chen, and B. Litkouhi, "Integrated estimation structure for the tire friction forces in ground vehicles," in Advanced Intelligent Mechatronics, 2016 IEEE Conference on. IEEE, 2016.

[29] E. Velenis, P. Tsiotras, C. Canudas-de Wit, and M. Sorine, "Dynamic tyre friction models for combined longitudinal and lateral vehicle motion," Vehicle System Dynamics, vol. 43, no. 1, pp. 3-29, 2005.

[30] E. Hashemi, A. Kasaiezadeh, S. Khosravani, A. Khajepour, N. Moshchuk, and S.-K. Chen, "Estimation of longitudinal speed robust to road conditions for ground vehicles," Vehicle System Dynamics, vol. 54, no. 8, pp. 1120-1146, 2016.

[31] C. Ahn, H. Peng, and H. Tseng, "Robust Estimation of Road Friction Coefficient," in American Control Conference (ACC), no. 3, 2011, pp. 3948-3953.

[32] R. Rajamani, G. Phanomchoeng, D. Piyabongkarn, and J. Y. Lew, "Algorithms for Real-Time Estimation of Individual Wheel Tire-Road Friction Coefficients," IEEE/ASME Transactions on Mechatronics, vol. 17, no. 6, pp. 1183-1195, 2012.

[33] M. Doumiati, A. Victorino, D. Lechner, G. Baffet, and A. Charara, "Observers for vehicle tyre/road forces estimation: Experimental validation," Vehicle System Dynamics, vol. 48, no. 11, pp. 1345-1378, 2010.

[34] W. Cho, J. Yoon, S. Yim, B. Koo, and K. Yi, "Estimation of tire forces for application to vehicle stability control," IEEE Transaction on Vehicular Technology, vol. 59, no. 2, pp. 638-649, 2010.

[35] J.-J. E. Slotine, W. Li, et al., Applied nonlinear control. prentice-Hall Englewood Cliffs, NJ, 1991, vol. 199, no. 1.

[36] H. K. Khalil, Nonlinear Systems, third ed. ed. Upper Saddle River, New Jersey: Prentice Hall, 2002.

[37] F. Garofalo, G. Celentano, and L. Glielmo, "Stability Robustness of Interval Matrices Via Lyapunov Quadratic Forms," IEEE Transactions on Automatic Control, vol. 38, no. 2, pp. 281-284, 1993.

[38] P. Gahinet, P. Apkarian, and M. Chilali, "Affine Parameter-Dependent Lyapunov Functions and Real Parametric Uncertainty," IEEE Transactions on Automatic Control, vol. 41, no. 3, pp. 436-442, 1996. 